\title{
Sexually transmitted organisms in sexually abused children
}

\author{
A J Robinson, J E M Watkeys, G L Ridgway
}

\begin{abstract}
Objective-To establish the prevalence of sexually transmitted organisms and other genital organisms in potentially sexually abused children.

Design-Prospective study of children attending an inner London department of community paediatrics for evaluation of possible sexual abuse.

Subjects-Children under 16 referred for evaluation of possible sexual abuse.

Outcome measures-Prevalence of sexually transmitted organisms in relation to age, symptoms, and type of abuse.

Results-Swabs were taken from 159 of 242 girls evaluated. The overall prevalence of sexually transmitted organisms was $3.7 \%$ : three girls were infected with gonorrhoea, four with Trichomonas vaginalis, and two with Chlamydia trachomatis. One girl had all three infections plus mycoplasmas. Mycoplasmas were identified in $22 \%$ of girls swabbed. Of 30 boys swabbed, none yielded a sexually transmitted organism.

Conclusions-There is a low prevalence of definitely sexually transmitted organisms in children who might have been abused. Other organisms possibly associated with sexual activity can be identified in this population. Screening for infection should be mandatory in presumed sexually abused girls with vaginal discharge and ideally should be undertaken in all children attending for evaluation of sexual abuse.

(Arch Dis Child 1998;79:356-358)
\end{abstract}

Centre, Mortimer

Market, Off Capper

Street, London

WC1E 6AU, UK

A J Robinson

Camden and Islington

Community Health

Services Trust,

Department of

Community

Paediatrics, 112

Hampstead Road,

London NW1 2LT, UK

J E M Watkeys

Department of

Microbiology,

University College

London Hospital,

London WC1E 6DB,

UK

G L Ridgway

Correspondence to: Dr Robinson.

Accepted 18 May 1998 compared the flora in girls with vulvovaginitis with those of a control group without addressing the possibility of sexual abuse. ${ }^{311}$ Other investigators have used control groups that did not exclude girls with a history of abuse ${ }^{4}{ }^{12}$; one study screened only for current sexual activity ${ }^{5}$; other studies had no control groups ${ }^{6}$; and in one study, three controls were reallocated to the abused group. ${ }^{13}$ In adults, some organisms have been shown to be associated with sexual activity, including mycoplasmas and organisms associated with bacterial vaginosis. ${ }^{10}$ However, the presence of mycoplasmas cannot be deemed to be diagnostic of sexual abuse because these organisms can be transmitted vertically and have been found in non-sexually active girls. ${ }^{10}$

There are inherent difficulties in interpreting studies identifying sexually transmitted organisms because it is difficult to eliminate child sexual abuse. It is not usually possible to obtain all necessary swabs from all children. This prospective study was undertaken to screen abused children for infection to determine the prevalence of sexually transmitted organisms in association with type of abuse and physical findings.

Subjects, methods, and results

Three hundred and nineteen children (77 boys, 242 girls) between the ages of 9 months and 16 years, attending an inner London paediatric department for evaluation of sexual abuse entered the study from July 1991 to July 1994. The diagnosis of child sexual abuse was based on the following: disclosure by the child; outcome of police and social services investigation; care and/or criminal proceedings; and the presence of physical findings supportive of abuse, such as hymenal tears. Swabs were taken of vaginal discharge (if present) or from the vaginal introitus, for Gram stain and culture for Neisseria gonorrhoeae, Chlamydia trachomatis, Trichomonas vaginalis, Candida spp, mycoplasmas, and other bacterial infections using standard methods. ${ }^{14}$ Bacterial vaginosis was diagnosed on two criteria, clinically abnormal vaginal discharge and clue cells on Gram stain. As prepubertal children have alkaline secretions, $\mathrm{pH}$ is invalid in this population as a diagnostic criterion for bacterial vaginosis. Anal swabs were taken from boys and girls reporting anal abuse, or with anal findings suggestive of abuse, for culture for $N$ gonorrhoeae and $C$ trachomatis. Urethral swabs were sent for isolation of $N$ gonorrhoeae and $C$ trachomatis. Blood tests for human immunodeficiency virus (HIV) and treponemal antibodies were performed at the request of parents or child in cases involving multiple abusers or when the perpetrator was known to be HIV positive. Genital warts were diagnosed clinically. 
Table 1 Organisms identified in girls swabbed (number swabbed)

\begin{tabular}{lllll}
\hline \multicolumn{3}{c}{ Age (years) } & & \\
\cline { 2 - 3 } Organisms & $\begin{array}{l}> \\
(n=42)\end{array}$ & $\begin{array}{l}3-10 \\
(n=117)\end{array}$ & $\begin{array}{l}>10 \\
(n=83)\end{array}$ & $\begin{array}{c}\text { Total } \\
(n=242)\end{array}$ \\
\hline Neisseria gonorrhoeae & $2(15)$ & $0(90)$ & $1(54)$ & $3(159)$ \\
Chlamydia trachomatis & $0(10)$ & $1(67)$ & $1(54)$ & $2(131)$ \\
Trichomonas vaginalis & $0(15)$ & $0(90)$ & $4(54)$ & $4(159)$ \\
Mycoplasmas & $0(8)$ & $6(42)$ & $15(46)$ & $21(96)$ \\
Candida albicans & $4(15)$ & $3(90)$ & $12(54)$ & $19(159)$ \\
Bacterial vaginosis & $0(15)$ & $1(90)$ & $2(54)$ & $3(159)$ \\
Anaerobes & $0(15)$ & $6(90)$ & $1(54)$ & $7(159)$ \\
Swabs unavailable & 27 & 27 & 29 & 83 \\
\hline
\end{tabular}

Swabs could not be taken from all sites in all children. Swabs were not taken from 83 girls and 47 boys: 49 children refused; 10 samples were toxic to culture; in the remainder there were no physical findings and either no evidence of sexual abuse that involved touching or penetration, or a delay of more than a year to presentation from time of abuse. Vaginal swabs were taken from 159 girls, and anal swabs from 63 children ( 28 boys and 35 girls); two boys had both urethral and anal swabs and two adolescent boys had only urethral swabs taken. Two throat swabs were taken from girls giving a history of oral sex.

Fifteen boys were aged under 3 years, 42 were between 3 and 10 years, and 20 were over 10 years of age. No sexually transmitted organisms were detected in the 28 boys who had anal swabs taken or in the four boys who had urethral swabs taken. Six of these 28 boys were aged under 3 years, three of whom were infected with $C$ albicans; 15 were between 3 and 10 years and seven were aged 10 years and older and they had no infections. Two boys were identified as abusers and had also been abused.

The infections identified according to the different age categories of girls are presented in table 1. Three girls (aged 11 months, $21 / 2$ years, and 13 years), all with vaginal discharge, were found to have gonorrhoea. Sexual contact was disclosed by the two oldest girls. Neither parent of the 11 month old girl had evidence of gonorrhoea and a neonatal infection screen, including eye swabs, was negative making vertical transmission unlikely. Two of 131 children screened for $C$ trachomatis were positive, one by direct immunofluorescence only. Both had evidence of penetrative abuse and one had vaginal discharge. $T$ vaginalis was found in four girls, all of whom were Afro-Caribbean, with oestrogenised hymena and vaginal discharge. One had anogenital warts. All had evidence of penetrative abuse (three hymenal tears, one enlarged hymenal opening, one previous termination of pregnancy).

Nineteen girls had candidal infection diagnosed on culture. Six of the younger children had a typical candidal nappy rash without laboratory confirmation. Mycoplasmas were identified in $21(22 \%)$ of the 96 girls for whom swabs were available (14 Ureaplasma urealyticum, four Mycoplasma hominis, three dual infection). Three girls were prepubertal and 15 had evidence of penetrative abuse. One girl disclosed digital abuse but her father admitted penile penetration.
A heavy growth of anaerobes was found in seven children. Five of the girls disclosed abuse involving touching of the genital area, one disclosed fellatio, and one child did not describe the type of abuse. Bacterial vaginosis was diagnosed in three girls, one of whom disclosed licking, one penetrative sex, and one unknown. Mobiluncus spp were identified on Gram stain of vaginal discharge in a 6 year old girl who alleged touching. Of these 11 girls, six had discharge, four had erythema alone, and one was asymptomatic. A girl aged 11 years and a boy aged 9 years had anogenital warts. None of six children tested serologically for syphilis and HIV were positive.

Other organisms identified included staphylococci, streptococci, Haemophilus influenzae, and Escherichia coli in eight girls aged 3-10 years, all of whom had vulvovaginitis.

Overall, seven of the 159 girls (3.7\%) had gonorrhoea, chlamydial infection, or trichomoniasis. One who was gang raped had all three infections plus mycoplasmas.

\section{Comments}

Our prevalence of $3.7 \%$ is similar to another British study. ${ }^{1}$ All the girls with gonorrhoea or trichomoniasis and two of three with chlamydial infection had vaginal discharge. The largest prospective study found $90 \%$ of girls with gonorrhoea and $33 \%$ of girls with $C$ trachomatis had vaginal discharge. ${ }^{2}$

These results support mandatory screening for $N$ gonorrhoeae, $C$ trachomatis, and $T$ vaginalis when there is a history of abuse and presence of vaginal discharge. Ideally, screening for these organisms should be included in any child sexual abuse investigation.

Mycoplasmas were present in $22 \%$ of girls swabbed in our study. All had either a history of or physical signs suggestive of penetrative sex. The identification of mycoplasmas cannot be deemed to be diagnostic of sexual abuse because they can be transmitted vertically and have been found in non-sexually active girls. Two reported studies comparing controls with sexually abused girls give conflicting conclusions on the significance of mycoplasmas in sexually abused children..$^{15} 16$ Further controlled studies are needed to assess the significance of mycoplasmas. Similarly, the significance of a heavy growth of anaerobes and organisms associated with bacterial vaginosis ${ }^{9}$ needs evaluation in relation to abuse. Finding suitable control groups for such studies poses ethical and practical difficulties, particularly in relation to consent, and matching for previous antibiotic use and age. Identification of organisms ( $N$ gonorrhoeae, $C$ trachomatis) using DNA amplification technology has been reported in prepubescent children using vaginal washings. ${ }^{17}$ Samples obtained non-invasively, such as urine, might be advantageous for screening abused children and might increase our understanding of genital infections in children. 
1 Hobbs CJ, Wynne JM. Sexual abuse of English boys and girls: the importance of anal examination. Child Abuse and girls: the importance of anal

2 Ingram DL, Everette VD, Liner PR, White ST, Rockwell LA. Epidemiology of adults sexually transmitted disease agents in children been evaluated for sexual abuse. Pediat Infect Dis f 1992;11:945-50.

3 Paradise JE, Campos JM, Friedman HM, Frismuth G. Vulvovaginitis in premenarcheal girls; clinical features and diagnostic evaluation. Pediatrics 1982;70:1028-31.

4 Hammerschlag MR, Albert S, Rosner I. Microbiology of the vagina in children: normal and potentially pathogenic organisms. Pediatrics 1978;62:57-62.

5 Schafer MA, Sweet RL, Ohm-Smith MJ, et al. Microbiology of the lower genital tract in postmenarcheal adolescent girls: differences by sexual activity, contraception and presence of non-specific vaginitis. $\mathcal{F}$ Pediatr 1985;107:974-81.

6 Fuster CD, Neinstein LS. Vaginal C. trachomatis prevalence in sexually abused girls. Pediatrics 1987;79:235-8.

7 White ST, Loda FA, Ingram DL, et al. Sexually transmitted diseases in sexually abused children. Pediatrics 1983;72:16disea 21 .

8 DeJong A. Sexually transmitted diseases in sexually abused children. Sex Transm Dis 1986;13:123-6.

9 Steele AM, de Snr Lazaro C. Transhymenal cultures for sexually transmissible organisms. Arch Dis Child 1994;71 423-7.
10 Holst E. Reservoir of four organisms associated with bacterial vaginosis suggests lack of sexual transmission. $f$ Clin Microbiol 1990;28:2035-9.

1 Heller RH, Joseph JM, Davis HJ. Vulvovaginitis in the premenarcheal child. F Paediatrics 1969;74:370-7.

12 Hammerschlag MR, Cummings M, Doraiswamy B, et al. Non-specific vaginitis following sexual abuse in children. Pediatrics 1985;75:1028-31.

13 Bartley D, Morgan L, Rimsza M. Gardnerella vaginalis in prepubertal girls. Am f Dis Child 1987;141:1014.

14 Stokes EJ, Ridgway GL, Wren MWD. Clinical microbiology. 7th ed. London: Arnold, 1993.

15 Hammerschlag MR, Doraiswamy B, Cox P, Cummings M, McCormack WM. Colonisation of sexually abused children with genital mycoplasmas. Sex Transm Dis 1987;14: $23-5$.

16 Ingram DL, White ST, Liner $\mathrm{P}$, et al. Ureaplasma urealyticum and large colony mycoplasma colonisation in female children and its relationship to sexual contact, age, and race. Child Abuse and Neglect 1993;16:265-72.

17 Embree JE, Lindsay D, Williams T, Peeling RW, Wood S, Morris M. Acceptability and usefulness of vaginal washes in premenarcheal girls as a diagnostic procedure for sexually transmitted diseases. Pediatr Infect Dis $\mathcal{F}$ 1996;15: $662-7$. 